\title{
A Grid Based Approach to Detect Mobile Target in Wireless Sensor Network
}

\author{
B. Anil Kumar ${ }^{1}$ and M. P. Srinivasa Rao ${ }^{2}$ \\ ${ }^{I}$ Department of Electronics and communication Engineering, GMR Institute of Technology, Rajam(A.P.)India. \\ ${ }^{2}$ Department of Physics, GMR Institute of Technology, Rajam(A.P.)India.
}

\begin{abstract}
The most prominent applications of wireless sensors networks are coverage, Target detection and field surveillance. This paper investigates detection of a target traversing the region being monitored by some number of sensors using minimum exposure path. We derive minimum exposure path formula from Integral geometry. We represent the sensor field as connected grid of points. Then minimum exposure is calculated for different grids of points. We consider the random and deterministic placement of sensors. It illustrates that the target detection can be achieved by choosing the appropriate number of sensors and grid of points.
\end{abstract}

Keywords: coverage, deployment, exposure, wireless sensor networks

\section{Introduction}

The rapid growth of wireless communication and embedded Micro-Electro-Mechanical Systems (MEMS) Technology has made wireless sensor networks possible [1]. These sensor networks are capable of observing the surroundings, processing the data and making decision based on the observations. Such network can be used to monitor the environment, detect, classify and locate specific events and track targets over a specific region. Examples of such systems are in surveillance, monitoring of pollution, Traffic, Agriculture or civil infrastructure [1]. Recently, a lot of research activities have been dedicated to sensor networks including Power management and life time of network $[6,15,16]$. Design issues related to the physical and media access layer[2,3] routing and transport protocols[4,5].

One of the fundamental issues in a wireless sensor network is the coverage (surveillance) problem. In general, this reflects how well an area is monitored or tracked by sensors. In the literature, this problem has been taken by in various ways. The Art Gallery Problem for example, deals with determining the number of observers necessary to cover an art gallery such that every point in the art gallery is monitored by at least one observer. This problem can be solved optimally in 2D plane [7]. Polynomial time algorithms to find the maximal breach path and maximal support path that are least and the best monitored in the sensor network are described in reference $[8,13]$. How to find the minimal and maximal exposure path that takes the duration that an object is monitored by sensors is addressed in [9]. Localized exposure based coverage and location discovery algorithms are proposed in [10].

This study investigates how well a target detection over a regions interest for a particular deployment of sensors. In order to detect a target moving through the region, sensors have to make local observations of the environment and collaborate to produce a global decision that reflects the status of the regions covered [11]. The collaboration requires local processing of the observations, communication between different nodes, and information fusion [12]. One possible measure for target detections in a sensor field is called path exposure. The path exposure is a measure of the likelihood of detecting a target traversing the region using a given path. The higher the path exposure, better the network deployment for target detection. The set of paths to be considered may be constrained by the environment. For example at a boarder battle field if a target is expected to be follow a road, only the paths consisting of the roads need to be considered.

The focus of this paper is to determine the minimum exposure path for target detection for a particular deployment of sensors in network field. If the minimum exposure value of a minimum exposure path is less, the target detection through that path is poor. For better detection the minimum exposure value must be as possible as high. In this study, the deployment of sensors is assumed in two cases. In the first case when the environment is unknown or hostile in which case the sensors may be air dropped or deployed by other means generally resulting in a random placement. On the other case predetermined placement when the environment is sufficiently known and under control, in which sensors can be strategically hand placed.

\section{Path Exposure}

Generally sensor nodes have different theoretical and physical characteristics, but most sensor nodes have the two common features (1) Sensing ability diminishes as distance increases and (2) Sensing ability can improve as the allotted sensing time increases. 
In view of these, for a sensor, we express the general sensitivity function $S(s, p)$ at any arbitrary point $p$ as

$$
S(s, p)=\frac{\lambda}{[d(s, p)]^{k}}
$$

Where $\mathrm{d}(\mathrm{s}, \mathrm{p})$ is the Euclidean distance between the sensor and the point $\mathrm{p}$, and the positive constants $\lambda$ and $\mathrm{k}$ are sensor technology dependent parameters. Depending on the environment the value k typically ranges from 1 to 4 [9].

Consider a rectangular sensor field $F$ with $n$ sensors deployed at locations $s_{i}, i=1,2, \ldots . . n$. . All sensor field intensity $\mathrm{I}(\mathrm{F}, \mathrm{p})$ for a point $\mathrm{p}$ in the field $\mathrm{F}$ is defined as the effective sensing measure at point $\mathrm{p}$ from all sensors in $\mathrm{F}$. Then $\mathrm{I}(\mathrm{F}, \mathrm{p})$ can express as

$$
I(F, p)=\sum_{1=1}^{n} S\left(s_{i}, p\right)(
$$

The path exposure is defined as an integral of a sensing function that generally depends on distance from sensors on a path from a starting point to destination point [9]. The exposure for an object in the sensor field during the interval $\left[t_{1}, t_{2}\right]$ along the path $p(t)$ is defined as

$$
E\left(p(t), t_{1}, t_{2}\right)=\int_{t_{1}}^{t_{2}} I\left(F, p(t)\left|\frac{d p(t)}{d t}\right| d t\right.
$$

where $\left|\frac{d p(t)}{d t}\right|$ is the element of arc or path length.

Using above formula, finding the exposure path in sensor networks field under arbitrary sensor deployment is difficult task. Therefore we present a very simple generic algorithm and several heuristics that can be used to obtain the solution to the exposure based coverage and surveillance.

The generic exposure problem domain is continuous and the exposure expression often does not have an analytical solution. To solve this problem we propose a simple algorithm that contains the following steps.

1. Transfer the continuous problem domain to discrete one.

2. Apply graph-theoretic abstraction.

3. Compute the minimum exposure path using Djikstra's shortest path algorithm

To transform the problem domain to the tractable discrete domain we use a generalized grid approach. For the sake of simplicity, we restrict our subsequent discussion to the 2-dimensions.

In the generalized grid based approach we divide the sensor network regions using an $n x n$ square grid. To simplify the problem further, the path is forced to exist only along the edges of the each grid square as shown in the Figure 1. We call the number of $n \times n$ square grids is the order of the grid. Since the minimal exposure path can travel in arbitrary direction through the sensor field, it is easy to see that the higher order grid creates significant change in the final results because it only allows horizontal and vertical movements. Hence 30x30 and 50x50 order grids considered. The minimum exposure path is then restricted to straight line edge segments of each square. Fig. 1 shows sensor field with 8 X8 grid order.

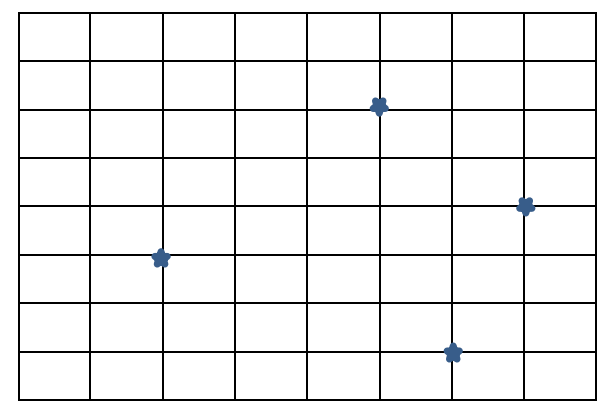

Figure. 1. Sensor field with 8x8 order grid and randomly deployed sensors $(\star \boldsymbol{k})$ 
We start our exposure calculation by considering the $n \times n$ square grid in the sensor field. Suppose there is only one sensor at point $(0,0)$, the sensitivity function at point $\mathrm{p}(\mathrm{x}, \mathrm{y})$ is given by using Equation(3)

$$
S(s(0,0), p(x, y))=\frac{1}{\left(\sqrt{x(t)^{2}+y(t)^{2}}\right)^{k}}
$$

The exposure along a path $\mathrm{p}(\mathrm{t})=(\mathrm{x}(\mathrm{t}), \mathrm{y}(\mathrm{t}))$ in the interval $[0,1]$ is

$$
E=\int_{0}^{1} \frac{1}{\left(\sqrt{x(t)^{2}+y(t)^{2}}\right)^{k}} \sqrt{\left(\frac{d x}{d t}\right)^{2}+\left(\frac{d y}{d t}\right)^{2}} d t
$$

This Integral calculates the exposure along the line segments of the grid elements. For the above Integral calculation we chose $\mathrm{k}=1$ (high power sensors) and 2, 3 (low power sensors). The above integral calculations serve as an analytic basis for the calculation of minimum exposure path in this paper.

The problem of finding the least exposure path from left side of the sensor field to right side of the sensor field is then equivalent to the problem of finding the least weight path from left side position to right side position on this grid. Such path can be efficiently determined using the Dijkstra's shortest path algorithm [9].

\section{Results and Discussions}

In the MATLAB (7.9) simulation, a wireless sensor network field is created. The sensor field in all cases is defined as a square, 1000 meters wide. We have assumed that the speed of the target is not a constant $\left(\left|\frac{d p(t)}{d t}\right| \neq 1\right)$. The exposure coefficient $\lambda$ is set to 1 , and field decay factor $(\mathrm{k})$ is taken in three cases as $\mathrm{k}=1,2$ and 3 . The sensing range of the sensors is considered as uniform and sensitivity value depends on field decay factor $(\mathrm{k})$ and point of observation in the field. The sensor always locates at center of its sensing field. The grid order in all cases is considered as 30x30 and 50x50. The results are averages on 10 randomly generated instances in random deployment.

\subsection{Random deployment of sensors}

In this random deployment various numbers of sensors are randomly deployed in the sensor field. The results in Table I show the average minimum exposure, average path length and grid order. From these tables we can observe wide range of minimum exposure paths from random deployment of sensors. This can be expected because of the random distribution of sensors. The number of sensors increases in the field, the minimum exposure value also increases whereas the grid order increases the minimum exposure value decreases. The tendency of these behavior represented in Fig. 2, 3 and 4. These results suggest that not only the number of sensors but also grid order effects the minimum exposure path. However the effect of grid order on minimum exposure path is less in high power sensor nodes $(\mathrm{k}=1)$, but remarkable impact on minimum exposure path in low power sensor nodes ( $\mathrm{k}=2$ and 4$)$.

In our results we have observed that as the number of sensors increase, the minimum exposure path length almost stable in all cases but in high power sensor field it is equal to the edge length and in low power $(\mathrm{k}=4)$ it approaching to the half of the perimeter value of the sensor field

TABLE I

Results of Randomly distributed sensors in network field (Average values for 10 sample values)

\begin{tabular}{|c|c|c|c|c|c|c|c|}
\hline \multirow{2}{*}{$\begin{array}{c}\text { Grid } \\
\text { order }\end{array}$} & \multirow{2}{*}{$\begin{array}{c}\text { Number } \\
\text { of sensors }\end{array}$} & $\begin{array}{c}\text { Average } \\
\text { Minimum } \\
\text { exposure }\end{array}$ & $\begin{array}{c}\text { Average Path } \\
\text { length } \\
\text { (meters) }\end{array}$ & $\begin{array}{c}\text { Average } \\
\text { Minimum } \\
\text { exposure }\end{array}$ & $\begin{array}{c}\text { Average } \\
\text { Path length } \\
\text { (meters) }\end{array}$ & $\begin{array}{c}\text { Average } \\
\text { Minimum } \\
\text { exposure }\end{array}$ & $\begin{array}{c}\text { Average } \\
\text { Path length } \\
\text { (meters) }\end{array}$ \\
\cline { 3 - 9 } & \multicolumn{2}{|c|}{$\mathrm{k}=1$} & \multicolumn{2}{|c|}{$\mathrm{k}=2$} & & \multicolumn{2}{c|}{$\mathrm{k}=4$} \\
\hline $30 \times 30$ & 12 & 36.808 & 1103.44 & 4.528 & 1544.82 & 0.0800 & 1724.13 \\
\hline $30 \times 30$ & 15 & 43.683 & 1096.55 & 5.390 & 1662.00 & 0.2627 & 1682.75 \\
\hline $30 \times 30$ & 20 & 60.410 & 1117.24 & 8.172 & 1551.70 & 0.3199 & 1779.31 \\
\hline $30 \times 30$ & 24 & 71.937 & 1075.86 & 10.869 & 1441.30 & 0.5380 & 1855.17 \\
\hline $30 \times 30$ & 30 & 94.440 & 1020.68 & 15.631 & 1531.03 & 0.6629 & 1813.79 \\
\hline $30 \times 30$ & 36 & 115.740 & 1041.37 & 17.605 & 1627.58 & 0.7352 & 1875.86 \\
\hline $50 \times 50$ & 12 & 34.710 & 1093.87 & 2.270 & 1697.9 & 0.0160 & 1771.42 \\
\hline $50 \times 50$ & 15 & 47.820 & 1069.38 & 3.727 & 1600.00 & 0.0422 & 1722.44 \\
\hline $50 \times 50$ & 20 & 65.127 & 1085.71 & 5.094 & 1563.30 & 0.0670 & 1773.46 \\
\hline $50 \times 50$ & 24 & 77.487 & 1065.30 & 6.426 & 1595.90 & 0.0936 & 1775.5 \\
\hline $50 \times 50$ & 30 & 95.530 & 1028.57 & 8.198 & 1416.30 & 0.1568 & 1944.48 \\
\hline $50 \times 50$ & 36 & 116.010 & 1032.65 & 11.007 & 1595.90 & 0.2155 & 1761.20 \\
\hline
\end{tabular}




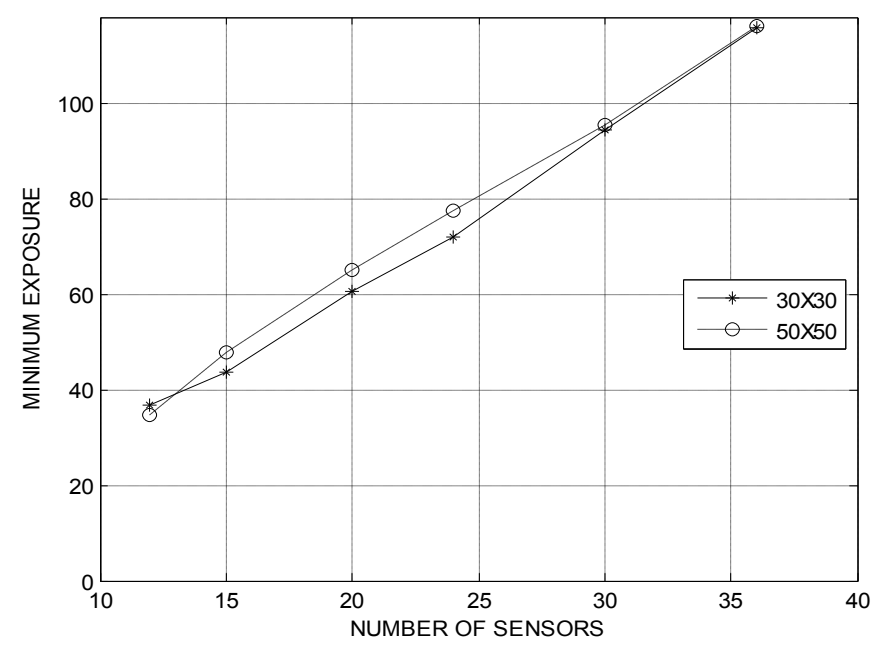

Figure. 2. Variation of Minimum exposure with number of sensors ( for $\mathrm{k}=1$ )

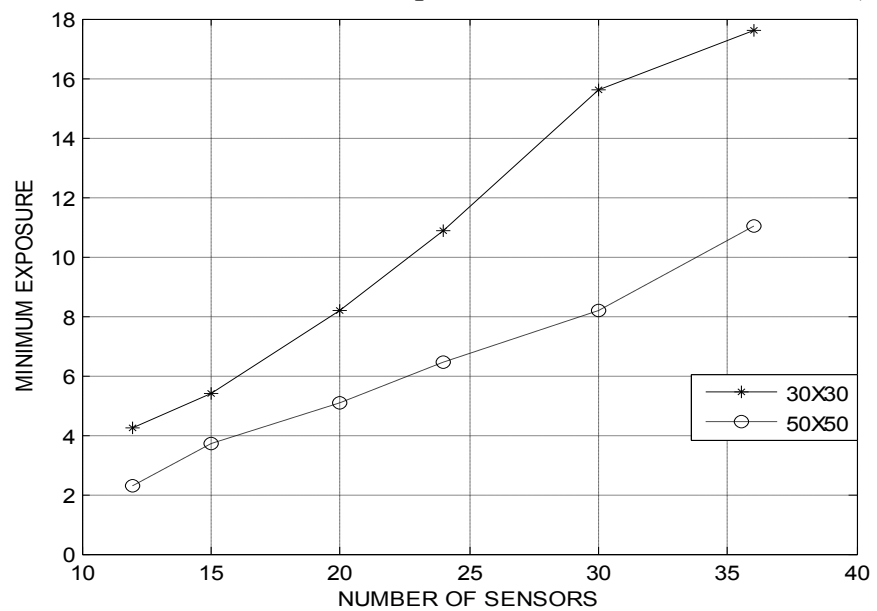

Figure. 3. Variation of Minimum exposure with number of sensors (for $\mathrm{k}=2$ )

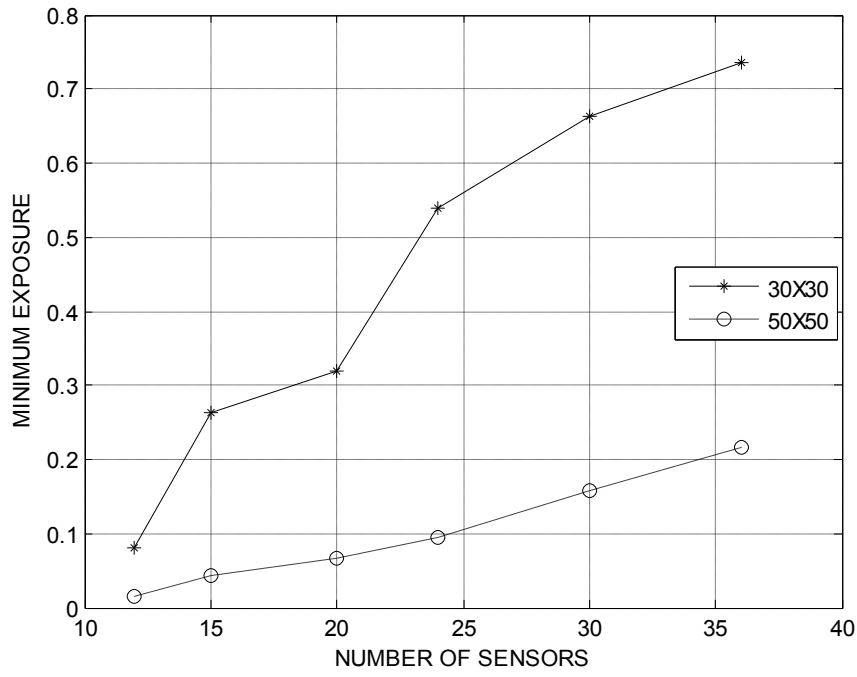

Figure. 4. Variation of Minimum exposure with number of sensors ( for $\mathrm{k}=4$ )

\subsection{Deterministic placement of sensors}

We have extended our study to the deterministic deployment of sensors. Table II lists the minimum exposure and path length for several sensor deployments using different sensors $(\mathrm{k}=1,2$ and 3$)$ and grid combinations. We have considered three types of sensor deployment schemes (1) Sensors are deployed along the diagonal lines of the sensor field i.e. (X) scheme,(2) sensors are placed along the bisector line of the faces of 
the field i.e. $(+)$ Scheme and (3) sensors are placed in a row along the middle of the field i.e. $(\|)$ scheme. The Fig. 5 and Fig.6 show the deployment of these three schemes.
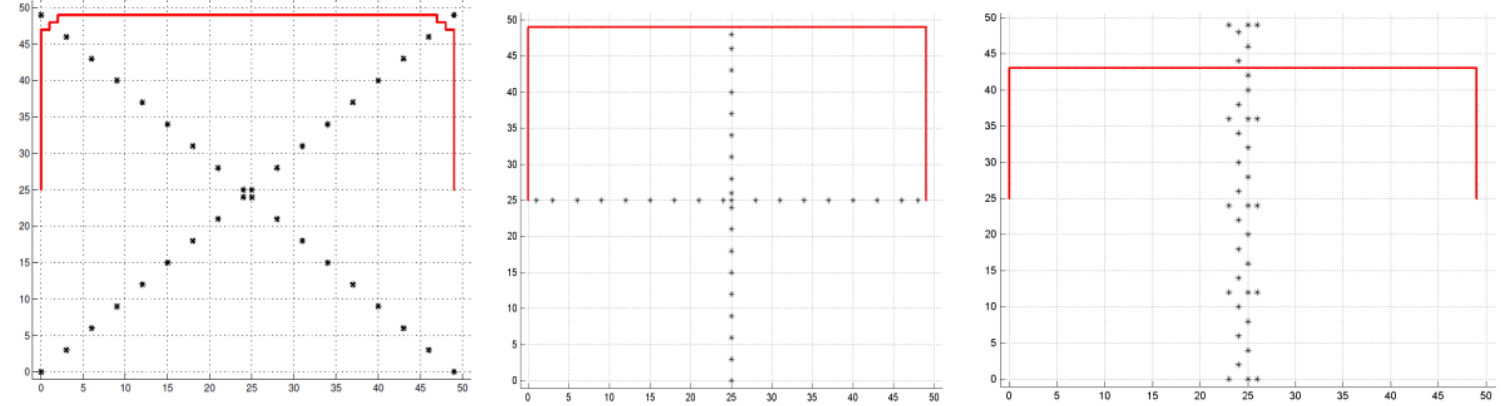

Figure 5. Minimum Exposure path using (X) Scheme, $(+)$ Scheme and $(\|)$ Scheme with 36 sensors for $k=4$ in grid order $50 \mathrm{X} 50$

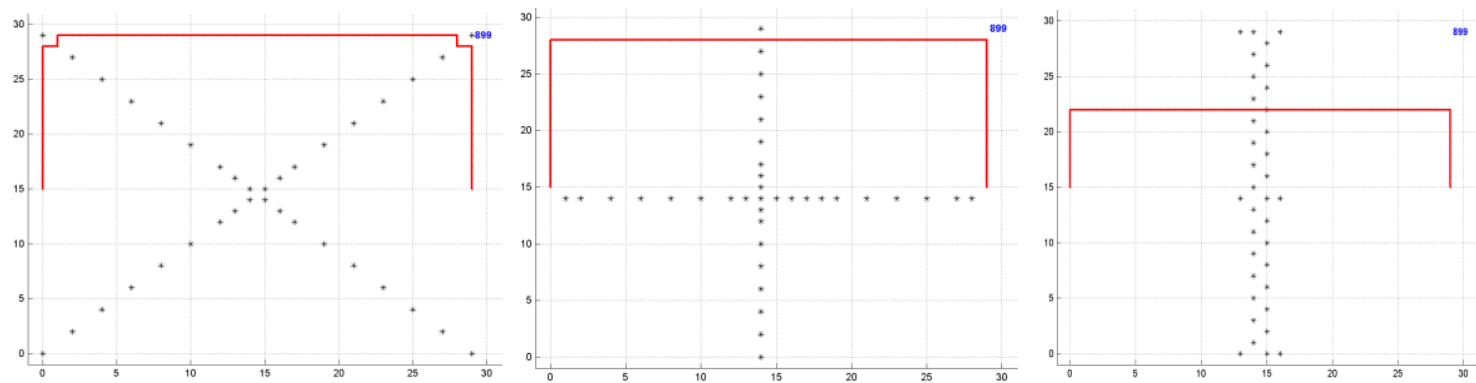

Figure 6. Minimum Exposure path using (X) Scheme, $(+)$ Scheme and $(\|)$ Scheme with 36 sensors for $k=4$ in grid order $30 \mathrm{X} 30$

In our results $(+)$ scheme of deployment provides best scheme forhigh minimum exposure in the sensor field $\mathrm{k}=1$ with grid order 50X50. In low power sensor field $(\mathrm{k}=2$ and 4$)$, the $(\|))$ scheme shows high exposure in the grid order 30X30. The scheme (X) shows medium exposure in the three deployment schemes and grid orders. Finally, the exposure along the minimum exposure path for all deployment schemes in deterministic deployment is higher than the randomly deployed schemes in the sensor field for grid order 30x30 and $50 \mathrm{X} 50$ Hence scheme of deployment and grid order depends upon the particular application.

TABLE II

Results of Deterministic placement of sensors in network field

\begin{tabular}{|c|c|c|c|c|c|c|c|c|}
\hline \multirow[t]{2}{*}{ Grid order } & \multirow[t]{2}{*}{$\begin{array}{l}\text { Number of } \\
\text { sensors }\end{array}$} & \multirow[t]{2}{*}{$\begin{array}{c}\text { Deployment } \\
\text { strategy }\end{array}$} & $\begin{array}{l}\text { Minimum } \\
\text { exposure }\end{array}$ & $\begin{array}{l}\text { Path length } \\
\text { (meters) }\end{array}$ & $\begin{array}{l}\text { Minimum } \\
\text { exposure }\end{array}$ & $\begin{array}{l}\text { Path length } \\
\text { (meters) }\end{array}$ & $\begin{array}{l}\text { Minimum } \\
\text { exposure }\end{array}$ & $\begin{array}{l}\text { Path length } \\
\text { (meters) }\end{array}$ \\
\hline & & & \multicolumn{2}{|c|}{$\mathrm{k}=1$} & \multicolumn{2}{|c|}{$\mathrm{k}=2$} & \multicolumn{2}{|c|}{$\mathrm{k}=4$} \\
\hline $30 \times 30$ & 12 & + & 44.54 & 1344 & 5.43 & 1758 & 0.417 & 1758 \\
\hline $30 \times 30$ & 12 & $\mathrm{x}$ & 33.17 & 1000 & 4.88 & 1482 & 0.131 & 1965 \\
\hline $30 \times 30$ & 12 & $\|$ & 42.57 & 1068 & 6.89 & 1551 & 1.612 & 1551 \\
\hline $30 \times 30$ & 20 & + & 76.02 & 1551 & 9.09 & 1965 & 0.741 & 1551 \\
\hline $30 \times 30$ & 20 & $\mathrm{x}$ & 71.06 & 1275 & 8.80 & 1965 & 0.464 & 1965 \\
\hline $30 \times 30$ & 20 & $\|$ & 69.55 & 1068 & 9.16 & 1965 & 1.824 & 1965 \\
\hline $30 \times 30$ & 36 & + & 138.92 & 1620 & 16.99 & 1965 & 3.638 & 1551 \\
\hline $30 \times 30$ & 36 & $\mathrm{x}$ & 132.02 & 1206 & 21.17 & 1965 & 5.104 & 1965 \\
\hline $30 \times 30$ & 36 & $\|$ & 124.16 & 1000 & 31.13 & 1344 & 10.44 & 1827 \\
\hline $50 \times 50$ & 12 & + & 48.82 & 1367 & 5.52 & 1775 & 1.599 & 1775 \\
\hline $50 \times 50$ & 12 & $\mathrm{x}$ & 33.22 & 1000 & 2.88 & 1000 & 0.027 & 1979 \\
\hline $50 \times 50$ & 12 & $\|$ & 42.91 & 1122 & 3.97 & 1897 & 0.259 & 1693 \\
\hline $50 \times 50$ & 20 & + & 81.63 & 1612 & 7.81 & 1489 & 1.700 & 1897 \\
\hline $50 \times 50$ & 20 & $x$ & 68.65 & 1122 & 5.09 & 2020 & 0.081 & 2020 \\
\hline $50 \times 50$ & 20 & $\|$ & 75.02 & 1122 & 7.63 & 1979 & 1.610 & 1979 \\
\hline $50 \times 50$ & 36 & + & 146.81 & 1979 & 13.32 & 1979 & 3.283 & 1979 \\
\hline $50 \times 50$ & 36 & $\mathrm{x}$ & 121.93 & 1040 & 13.62 & 1979 & 1.221 & 1979 \\
\hline $50 \times 50$ & 36 & $\|$ & 121.95 & 1000 & 14.94 & 1816 & 3.326 & 1734 \\
\hline
\end{tabular}




\section{Conclusions}

This paper addresses the coverage or surveillance problem in the wireless sensor field. A mathematical formula is derived for exposure along the grid paths. Based on the above formula the minimum exposure is calculated for various sensor deployments in the sensor field. The minimum exposure is used as a measure of the deployment of the sensors and the goal being to maximize the exposure of the minimum exposure path to detect the target in the sensor field. We found from our results that minimum exposure influenced by both number of sensors and grid order. Low power sensor placement is much effected by both its deployment and grid order. The above results indicate that for the right deployment of sensors sensor power and grid order to be considered.

\section{Acknowledgement}

The authors would like to thank GMR Institute of Technology, Rajam for proving constant encouragement and support.

\section{References}

[1] I. F. Akyildiz et al., “A Survey on Sensor Networks”, IEEE Comm.Mag., 40(8): Aug. 2002.pp .102-116

[2] E. Shih, S.-H. Cho, N. Ickes, R. Min, A. Sinha, A. Wang and A.Chandrakasan. "Physical layer driven protocol and algorithm design forenergy-efficient wireless sensor networks", in: ACM Int'l Conf. on Mobile Computing and Networking (MobiCom) (2001) pp. $272-287$.

[3] W. Ye, J. Heidemann and D. Estrin, “An energy-efficient MAC protocol for wireless sensor networks”, in: IEEE INFOCOM (2002) pp. 1567-1576.

[4] D. Ganesan, R. Govindan, S. Shenker and D. "Estrin, Highly resilient, energy efficient multipath routing in wireless sensor networks", ACM Mobile Comput. andCommun. Review 5(4) (2001) pp.11-25.

[5] W.R. Heinzelman, A. Chandrakasan and H. Balakrishnan, "Energyefficient communication protocols for wireless microsensor networks", in: Hawaii Int'l Conf. on Systems Science (HICSS) (2000).

[6] M. Cardei and D.-Z. Du, "Improving wireless sensor network lifetime through power aware organization," Wireless Netw., vol. 11, no. 3, (2005) pp.333-340,

[7] J. O'Rourke, Computational geometry column 15, Int'1 Journal of Computational Geometry and Applications 2(2) (1992),pp. 215217.

[8] S. Meguerdichian, F. Koushanfar, M. Potkonjak and M.B. Srivastava, "Coverage problems in wireless ad-hoc sensor networks". in: IEEE INFOCOM (2001) pp. 1380-1387.

[9] S. Meguerdichian, F. Koushanfar, G. Qu and M. Potkonjak, Exposure in wireless ad-hoc sensor networks, in: $A C M$ Int'l Conf. on MobileComputing and Networking (MobiCom) (2001) pp. 139-150

[10] S. Meguerdichian, S. Slijepcevic, V. Karayan and M. Potkonjak, Localized algorithms in wireless ad-hoc networks: location discovery and sensor exposure, in: ACM Int'l Symp. on Mobile Ad Hoc Networkingand Computing (MobiHOC) (2001) pp. 106116 .

[11] R. R. Brooks and S. S. Iyengar. Multi-Sensor Fusion:Fundamentals and Applications with Software. PrenticeHall, 1998.

[12] P. Varshney. Distributed Detection and Data Fusion.Springer-Verlag New-York, 1996.

[13] L. Lazos, R. Poovendran, and J. Ritcey, "Detection of mobile targets on the plane and in space using heterogeneous sensor networks," WirelessNetw., vol. 15, no. 5,(2009) pp. 667-690..

[14] EdoardoAmaldi, Antonio Capone, MatteoCesana, and IlarioFilippini, "Design of Wireless Sensor Networks for Mobile Target Detection", IEEE/ACM transactions on networking, vol. 20, no. 3, june (2012) pp.784-797

[15] H. Liu, X. Jia, P. Wan, C. Yi, S. Makki, and N. Pissinou, "Maximizing lifetime of sensor surveillance systems," IEEE/ACM Trans.Netw., vol. 15, no. 2, 2007.

[16] Carlos T. Calafate, Carlos Lino, Arnoldo Diaz-Ramirez, Juan-Carlos Cano and Pietro Manzoni “An Integral Model for Target Tracking Based on the Use of a WSN" Sensors 2013, Vol.13, pp.7250-7278 